\title{
Tri-virus/GD2-specific Allogeneic Cytotoxic T-lymphocytes
}

National Cancer Institute

\section{Source}

National Cancer Institute. Tri-virus/GD2-specific Allogeneic Cytotoxic T-lymphocytes. NCI

Thesaurus. Code C99132.

Allog eneic tri-viral specific, Epstein-Barr virus (EBV), cytomegalovirus (CMV) and adenovirus (Ad), cytotoxic T-lymphocytes (tV-CT Ls) expressing a chimeric antigen receptor (CAR) specific for disialog ang lioside GD2 with potential antineoplastic activity. Tri-virus/GD2-specific allogeneic CT Ls are produced by transducing tV-CT Ls with a GD2specific CAR retroviral vector. Upon administration, after an allogeneic hematopoietic stem cell transplant, these CT Ls may be selective towards EBV, CMV, and Ad-infected cells and GD2-expressing tumor cells. The human glycosphingolipid GD2 is a tumor associated antigen overexpressed on the surface of all tumors of neuroectodermal origin. 